\title{
Comparison of order reduction algorithms for application to electrical networks
}

\author{
Lj. Radić-Weissenfeld ${ }^{1,2}$, S. Ludwig ${ }^{1,2}$, and W. Mathis ${ }^{1}$ \\ ${ }^{1}$ Leibniz University Hannover, Institute of Electromagnetic Theory, Hannover, Germany \\ ${ }^{2}$ Fraunhofer Research Institution for Electronic Nanosystems, Advanced System Engineering, Paderborn, Germany
}

\begin{abstract}
This paper addresses issues related to the minimization of the computational burden in terms of both memory and speed during the simulation of electrical models. In order to achieve a simple and computational fast model the order reduction of its reducible part is proposed. In this paper the overview of the order reduction algorithms and their application are discussed.
\end{abstract}

\section{Introduction}

Electrical models suggested for order reduction are electrical networks that represent the electromagnetic behaviour and performance of IC interface, electrical representation of signal integrity or power integrity. The electrical representations are huge electrical networks especially if they describe complex structures up to frequencies in the giga range. Therefore smaller electrical networks are desired in order to minimize computational costs in terms of both memory and speed during time and frequency simulation.

There are several ways to reach a smaller model. A smaller model can be created in the modelling process by considering restricted properties of a restricted interface (Kazemzadeh et al., 2008). If the model precisely describes properties of the whole IC interface for a high frequency range the smaller model can be obtained by utilizing order reduction algorithms (Antoulas and Sorensen, 2001; Ludwig et al., 2008; Ludwig and Mathis, 2009; Odabasioglu et al., 1998). Since created electrical models in the modeling phase are passive electrical networks, which usually consist of only time invariant elements, the order reduction algorithms based on the use of the Krylov subspace and Lyapunov balancing are suggested. These order reduction algorithms reduce the num-

\section{Correspondence to:}

Lj. Radić-Weissenfeld

(radic@tet.uni-hannover.de) ber of unknowns i.e. degrees of freedom as much as possible without sacrificing accuracy at the observation points.

In this paper order reduction algorithms based on the system projection on the Krylov subspace and Lyapunov balancing are compared. Moreover the prerequisite for combining these order reduction algorithms is discussed. The focus is particularly on the multipoint frequency-domain projection of the original model on the Krylov subspace, projectionbased truncated balanced method and the combination of those.

\section{Description of electrical models}

Electrical models are defined either as single-input singleoutput (SISO) systems or as multiple-input multiple-output (MIMO) systems. Systems are parts of a model connected to the input/output ports. Therefore inputs and outputs of a system are ports of an electrical model and a system is a reducible part of an electrical model as it is shown in Fig. 1.

The electrical model in Fig. 1 can be described by the following system equations

$$
\begin{aligned}
\mathbf{C s} \mathbf{X} & =\mathbf{G X}+\mathbf{B U} \\
\mathbf{V} & =\mathbf{L X},
\end{aligned}
$$

where $\mathbf{U}, \mathbf{V} \in \mathbb{R}^{p}$ are input and output vectors, $p$ is the number of ports, while $\mathbf{C}, \mathbf{G} \in \mathbb{R}^{N \times N}, \mathbf{B}, \mathbf{L}^{T} \in \mathbb{R}^{N \times p}$ are system matrices of the order $N$. The matrices and vectors in Eq. (2) result from the modified nodal analysis of the electrical network.

After the order reduction, order reduced system matrices $\mathbf{C}_{\text {red }}, \mathbf{G}_{\text {red }} \in \mathbb{R}^{q \times q}, \mathbf{B}_{\text {red }}, \mathbf{L}_{\text {red }}^{T} \in \mathbb{R}^{q \times p}$ are of the order $q \ll N$, while the order reduced model has the same number of inputs and outputs $p$. Now the order reduced system is described by following equations

$$
\begin{aligned}
\mathbf{C}_{\mathrm{red}} s \mathbf{X}_{\text {red }} & =\mathbf{G}_{\text {red }} \mathbf{X}_{\text {red }}+\mathbf{B}_{\text {red }} \mathbf{U} \\
\widetilde{\mathbf{V}} & =\mathbf{L}_{\text {red }} \mathbf{X}_{\text {red }},
\end{aligned}
$$




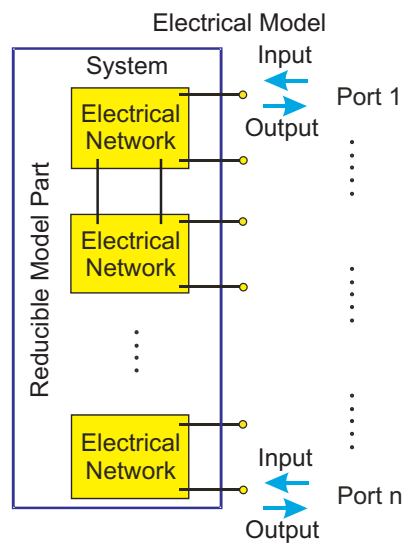

Fig. 1. A block diagram of an electrical model.

After the order reduction vector $\mathbf{V}$ is slightly changed and in Eq. (3) $\widetilde{\mathbf{V}} \approx \mathbf{V}$ holds. The change of the output vector emerges because the vector depends on the system. Therefore the change of the vector is a result of the order reduction of the system. Figure 2 illustrates the consequences of the order reduction of a linear system. The only difference between the original and reduced system is their description by system matrices, while input and output vectors are almost preserved.

The order reduced system can be synthesized as a reduced electrical model for time and frequency analyses and simulations with a circuit simulator (Ludwig et al., 2008; Yang et al., 2007).

\section{Order reduction algorithms}

The combination of order reduction algorithms, derived from a Krylov subspace projection and singular value based methods are successfully used for the order reduction of passive linear electrical networks (Antoulas and Sorensen, 2001). The Krylov subspace projection method is commonly used for models with a low number of input/output ports and a high order. The singular value based methods are successfully used for models of a modest order, i.e. a few hundred and a high number of ports.

\subsection{Krylov-subspace projection}

The basic principle of order reduction algorithms based on the moment matching is the projection of the system on the Krylov subspace (Odabasioglu et al., 1998; Freund, 2004). These order reduction algorithms are based on a mere projection utilizing the projection matrix that contains only the most dominant system part. Since only the most dominant system part is projected, the created system is small having the same dominant properties as the original. The Krylov
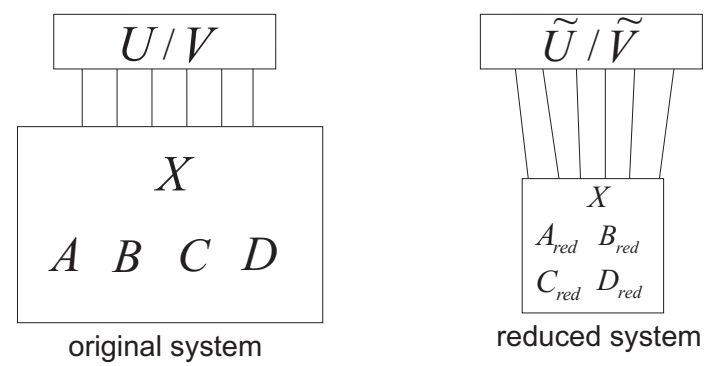

Fig. 2. A block diagram of an original and order reduced system.

subspace used in the order reduction is generated by matrices from Eq. (2)

$$
\begin{array}{r}
K r(\mathbf{R}, \mathbf{E}, q) \equiv \operatorname{colsp}\left[\mathbf{R}, \mathbf{A} \mathbf{R}, \mathbf{A}^{2} \mathbf{R}, \ldots,\right. \\
\left.\mathbf{A}^{k-1} \mathbf{R}, \mathbf{A}^{k} \mathbf{r}_{0}, \mathbf{A}^{k} \mathbf{r}_{1}, \ldots, \mathbf{A}^{k} \mathbf{r}_{l}\right],
\end{array}
$$

where $k=\lfloor q / N\rfloor$ and $l=q-k N$ (Odabasioglu et al., 1998). $q$ is the order to which the system of the order $N$ is reduced. The order reduction is based on the moment matching, i.e. preserving the coefficients of the Taylor expansion of a transfer function

$\mathbf{Y}(s)=\mathbf{M}_{0}+\mathbf{M}_{1} s+\mathbf{M}_{2} s^{2}+\cdots$,

where $\mathbf{M}_{k} \in \mathbb{R}^{N}$ is a matched moment. The system matrices are reduced, while preserving the passivity of the reduced system (Odabasioglu et al., 1998). The similarity between original and order reduced system depends on the selected expansion points as well as on the dimension of the Krylov subspace. This order reduction does not enable neither an error prediction nor the preservation of the dominant controllability and observability system part.

\subsection{Lyapunov balancing for order reduction}

Another group of order reduction algorithms is built by algorithms based on the dominant controllability and observability system part. The controllability and observability of a system is defined by controllability $\mathbf{P}$ and observability $\mathbf{Q}$ Gramians as solutions of the Lyapunov equations

$$
\begin{aligned}
\mathbf{G P C}+\mathbf{C P A}^{T}+\mathbf{B B}^{T} & =\mathbf{0} \\
\mathbf{C Q G}+\mathbf{G}^{T} \mathbf{Q C}+\mathbf{L L}^{T} & =\mathbf{0},
\end{aligned}
$$

where $\mathbf{G}, \mathbf{B}, \mathbf{L} \in \mathbb{R}^{N \times N}$ are state space matrices of an original system given in Eqs. (1) and (2).

The order reduction of system described by Eq. (6) can be done by truncation of the weak system part recognized in small singular values of the product of observability and controllability Gramians (Antoulas and Sorensen, 2001). These singular values can be used to determine the error of the order reduction as shown in (Obinata and Anderson, 2001; Kokotović et al., 1976) or to indicate the convergence of the absolute magnitude error as in Phillips and Silveira (2005). 


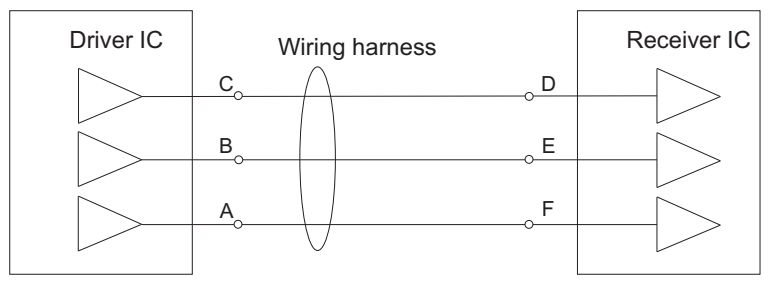

Ground plane

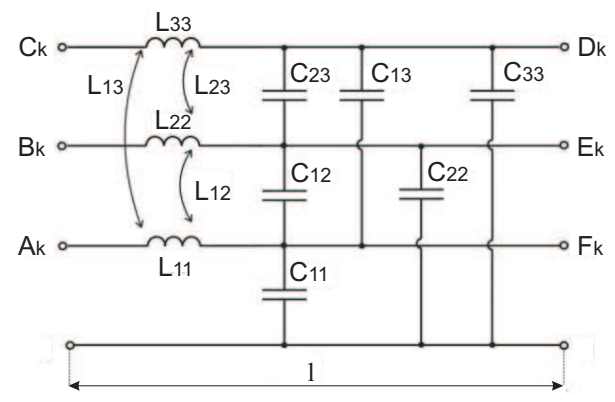

Fig. 4. Per-unit-length cable harness model (Musolino, 2007).
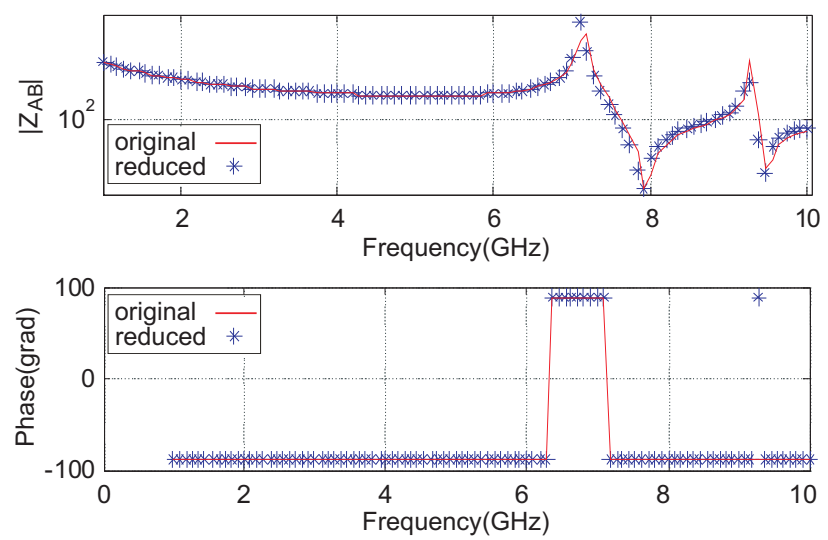

Fig. 5. Amplitude and phase of original and reduced impedance $Z_{A B}$, between ports $A$ and $B$.

erties of the original system in the whole frequency range of interest is shown in Figs. 5 and 6. The magnitude and phase of the original and order reduced transfer functions are very similar, since good expansion points are selected. Further order reduction based on Lyapunov equations (Eq. 6) is not suggested, because the whole redundant system part is rejected as well as a part of the system that can be defined as a dominant part. This is supported by results gained from the order reduction by using two expansion points and twodimensional Krylov subspace. In this case the system of the order 24 does not approximate the original system at all, because the dominant system part is rejected. Indeed, Fig. 7 shows that beside the first 36 singular values the following singular values can be considered as related to the dominant system part. The difference between the magnitude of the 4th and 24th singular values is as big as the difference between the 24th and 43th singular values. Therefore the additional order reduction would reduce the system part of importance and using more expansion points would not drastically reduce the error of the order reduction.

While the original system has sparse matrices, the order reduced system is described by dense matrices. The density 

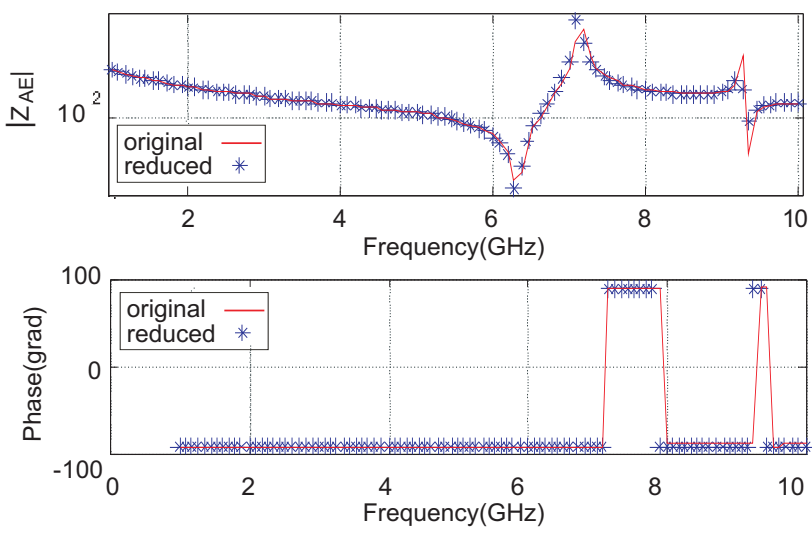

Fig. 6. Amplitude and phase of original and reduced impedance $Z_{A E}$, between ports $A$ and $E$.

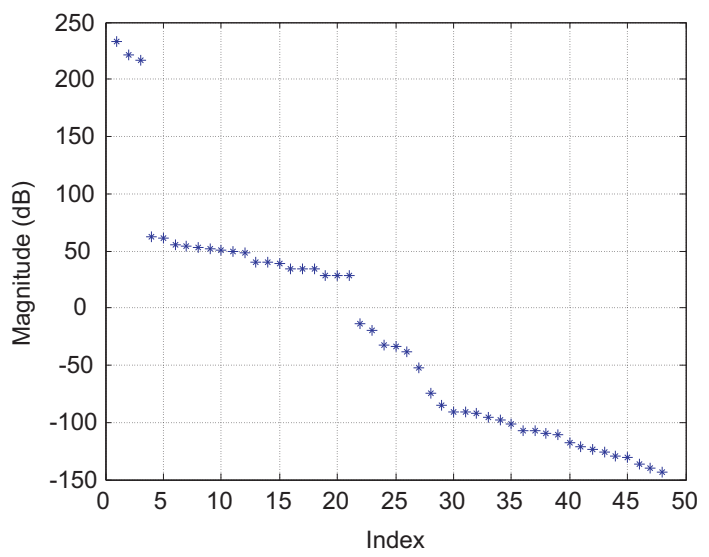

Fig. 7. Singular values of the system.

of the order reduced matrices influences the duration of the time simulation. This is due to the fact that the electrical circuit build from the dense matrices has the number of the nodes equal to the matrix size and it is almost fully connected network, if the synthesis method from Ludwig et al. (2008); Yang et al. (2007) is utilized.

\section{Conclusions}

In this paper an overview of order reduction techniques for electrical networks is given. The most successfully applied order reduction algorithm is a mere projection of a system on the Krylov subspace. Often this algorithm is used as a first step of the order reduction. Further order reduction can be done if the projection does not select a whole redundant system part. For the implementation of the second-step of the algorithm the Lyapunov balanced based algorithm is suggested, which selects the redundant system part.
Acknowledgements. Represented research and development work is carried out in the frame of the MEDEA+ project A701 PARACHUTE project (Parasitic Extraction and Optimization for Efficient Microelectronic System Design and Application). This particular research was supported by the BMBF (Bundesministerium fuer Bildung und Forschung) Federal Republic of Germany under 01M 3169 A, 01M $3169 \mathrm{D}$ and 01M $3169 \mathrm{E}$. The responsibility for this publication is held by authors only.

\section{References}

Antoulas, A. C. and Sorensen, D. C.: Lyapunov, Lanczos, and Inertia, Linear Algebra and its Applications, 326, 137-150, 2001.

Freund, R. W.: SPRIM: structure-preserving reduced-order interconnect macromodeling, Proceedings of the IEEE/ACM International conference on Computer-aided design, pp. 80-87, 2004.

Kazemzadeh, R., Ludwig, S., Radić-Weissenfeld, Lj., and Mathis, W.: Efficient Modelling of IC Conducted Emission for Power Integrity Analysis, Adv. Radio Sci., in press, 2009.

Kokotović, P. V., O’Malley, R. E., and Sannuti, P.: Singular perturbation and order reduction in control theory - An overview, Automatica, 12, 123-132, 1976.

Ludwig, S., Radić-Weissenfeld, Lj., Mathis, W., and John, W.: Efficient Model Reduction of Passive Electrical Networks with a Large Number of Independent Sources, Proceedings of the IEEE International Symposium on Circuit and Systems - ISCAS, pp. 1280-1283, Seattle, USA, 2008.

Ludwig, S. and Mathis, W.: Transformation of Passive Electrical Networks with Distributed PWL-Sources using a Model Order Reduction, Adv. Radio Sci., in press, 2009.

Musolino, F.: EFT immunity characterization of ICs, Proceedings of the EMC Compo, pp. 163-167, 2007.

Obinata, G. and Anderson, B. D. O.: Model Reduction for Control System Design, Springer-Verlag, London, Great Britain, 2001.

Odabasioglu, A., Celik, M., and Pillegi, L.: PRIMA: Passive Reduced-Order. Interconnect Macromodeling Algorithm, IEEE Transactions on computer-aided design of integrated circuits and systems, 17(8), 645-654, 1998.

Phillips, J. R. and Silveira, M.: Poor Man's TBR: A Simple Model Reduction Scheme, IEEE Transactions on Computer-Aided Design of Integrated Circuits and Systems, 24(1), 43-55, 2005.

Radić-Weissenfeld, Lj., Ludwig, S., Mathis, W., and John, W.: Model order reduction of linear time invariant systems, Adv. Radio Sci., 6, 129-132, 2008a, http://www.adv-radio-sci.net/6/129/2008/.

Radić-Weissenfeld, Lj., Ludwig, S., Mathis, W., and John, W. Two-step Order Reduction of IC Conducted Emission Models, Asia-Pacific EMC Symposium, Singapur, 2008b.

Radić-Weissenfeld, Lj., Ludwig, S., Mathis, W., and John, W.: Comparison of order reduction algorithms for IC emission models, EMC Symposium, Wroclaw, 2008c.

Yang, F., Zeng, X., Su, Y., and Zhou, D.: RLCSYN: RLC Equivalent Circuit Synthesis for Structure-Preserved Reduced-order Model of Interconnect, IEEE International Symposium on Circuit and Systems - ISCAS, New Orleans, USA, 2007. 